\title{
Manufactured solutions and the verification of three-dimensional Stokes ice-sheet models
}

\author{
W. Leng ${ }^{1}$, L. Ju ${ }^{2,3}$, M. Gunzburger ${ }^{4}$, and S. Price ${ }^{5}$ \\ ${ }^{1}$ State Key Laboratory of Scientific and Engineering Computing, Chinese Academy of Sciences, Beijing 100190, China \\ ${ }^{2}$ Department of Mathematics, University of South Carolina, Columbia, SC 29208, USA \\ ${ }^{3}$ Beijing Computational Science Research Center, Beijing 100084, China \\ ${ }^{4}$ Department of Scientific Computing, Florida State University, Tallahassee, FL 32306, USA \\ ${ }^{5}$ Theoretical Division, Los Alamos National Laboratory, Los Alamos, NM 87545, USA
}

Correspondence to: L. Ju (ju@math.sc.edu) and M. Gunzburger (gunzburg@ @fsu.edu)

Received: 9 June 2012 - Published in The Cryosphere Discuss.: 20 July 2012

Revised: 24 November 2012 - Accepted: 28 November 2012 - Published: 4 January 2013

\begin{abstract}
The manufactured solution technique is used for the verification of computational models in many fields. In this paper, we construct manufactured solutions for the threedimensional, isothermal, nonlinear Stokes model for flows in glaciers and ice sheets. The solution construction procedure starts with kinematic boundary conditions and is mainly based on the solution of a first-order partial differential equation for the ice velocity that satisfies the incompressibility condition. The manufactured solutions depend on the geometry of the ice sheet, basal sliding parameters, and ice softness. Initial conditions are taken from the periodic geometry of a standard problem of the ISMIP-HOM benchmark tests. The upper surface is altered through the manufactured solution procedure to generate an analytic solution for the timedependent flow problem. We then use this manufactured solution to verify a parallel, high-order accurate, finite element Stokes ice-sheet model. Simulation results from the computational model show good convergence to the manufactured analytic solution.
\end{abstract}

\section{Introduction}

Model verification and validation are crucial steps in the development and testing of computational models. Verification is the process of determining if a particular implementation and solution of a given mathematical model (for example through some choice of model discretization and numerical solution algorithms) is complete and error free. Validation aims to answer the entirely separate question of whether or not a given mathematical model is an accurate representation of the real-world process it aims to mimic. Manufactured analytical solutions provide one means for performing model verification.

The goal of this paper is to provide the means, through the use of manufactured solutions, for the verification of threedimensional ice-sheet models as a necessary step for providing accurate, science-based predictions of ice-sheet changes over climatic time scales. Manufactured analytical solutions have been used previously by the ice-sheet modeling community (Bahr, 1996; Bueler et al., 2005, 2007; Sargent and Fastook, 2010).

The nonlinear, three-dimensional (3-D) Stokes model, which does not utilize any approximations based on the shallowness of the domain, is the standard non-shallow description of ice flows within glaciers and ice sheets (Le Meur et al., 2004; Leysinger Vieli et al., 2004; Gagliardini and Zwinger, 2008; Burstedde et al., 2009; Zhang et al., 2011; Larour et al., 2012; Leng et al., 2012). The more commonly used shallow-ice, shallow-shelf, L1L2, and first-order approximations are reduced forms of the 3-D Stokes model that are numerically simpler and computationally cheaper to solve, but with an attendant loss of fidelity in some situations (see discussions in Dukowicz et al. (2010); Schoof and Hindmarsh (2010) and the references cited therein). As an example, the ISMIP-HOM project (Pattyn et al., 2008) compared diagnostic output from a number of "higher-order" (i.e., those accounting for horizontal stress gradients) and 
Stokes flow ice-sheet models on idealized domains. For certain combinations of domain aspect ratio, basal roughness, and basal sliding conditions, higher-order and Stokes model solutions differ significantly. Ideally, diagnostic output from Stokes models should first be compared with that from lowerorder approximations in order to identify portions of the model domain for which the Stokes (relatively expensive) versus the reduced (relatively cheaper) sets of equations apply with sufficient accuracy (e.g., Morlighem et al., 2010). Such an approach would allow for an ideal tradeoff between model accuracy and expense (e.g., Seroussi et al. (2012)).

Manufactured solutions for the verification of isothermal Stokes ice-sheet models were recently proposed by Sargent and Fastook (2010) for two and three-dimensional model domains. In their clever approach, which we follow here, the major task in the construction of the analytic solution is to solve a first-order partial differential equation. However, due to an error in their solution method for this key part in the three-dimensional case, the Sargent and Fastook (2010) manufactured solutions (both the general form of the solution and the solution for the specific geometry) for the 3-D Stokes model are incorrect. In Leng et al. (2012), the authors "extruded" the two-dimensional analytical solution of Sargent and Fastook (2010) to a third dimension and used it to verify the output from their Stokes ice-sheet model. This method of generating and applying a 3-D manufactured solution is far from optimal, as the 3-D model is applied in a 2-D mode, leaving parts of the 3-D model untested. In this paper, we rectify this deficiency by generating fully $3-\mathrm{D}$ manufactured solutions for the validation of isothermal, nonlinear Stokes models of ice flow.

The paper is organized as follows. In Sect. 2, we present the 3-D, nonlinear Stokes equations for modeling isothermal ice sheets along with some related boundary conditions. In Sect. 3, we correct the errors made by Sargent and Fastook (2010) and derive in detail the correct manufactured analytical solutions for the isothermal, 3-D, time-dependent Stokes ice-sheet model. In Sect. 4, we use the manufactured solutions for the numerical verification of the parallel finite element ice-sheet model of Leng et al. (2012). Finally, in Sect. 5, we provide some concluding remarks.

\section{Governing equations of the Stokes ice-sheet model}

\subsection{Ice dynamics and evolution}

The dynamical behavior of ice sheets is modeled by the Stokes equations for an incompressible, power-law viscous fluid. Letting $\left[0, t_{\max }\right]$ denote the time interval of interest and $\Omega_{t}$ the three-dimensional spatial domain occupied by the ice sheet, we have

$$
\frac{\partial\left(2 \mu \frac{\partial u}{\partial x}-p\right)}{\partial x}+\frac{\partial\left(\mu\left(\frac{\partial u}{\partial y}+\frac{\partial v}{\partial x}\right)\right)}{\partial y}+\frac{\partial\left(\mu\left(\frac{\partial u}{\partial z}+\frac{\partial w}{\partial x}\right)\right)}{\partial z}=0,
$$

$$
\begin{aligned}
& \frac{\partial\left(\mu\left(\frac{\partial u}{\partial y}+\frac{\partial v}{\partial x}\right)\right)}{\partial x}+\frac{\partial\left(2 \mu \frac{\partial v}{\partial y}-p\right)}{\partial y}+\frac{\partial\left(\mu\left(\frac{\partial v}{\partial z}+\frac{\partial w}{\partial y}\right)\right)}{\partial z}=0, \\
& \frac{\partial\left(\mu\left(\frac{\partial u}{\partial z}+\frac{\partial w}{\partial x}\right)\right)}{\partial x}+\frac{\partial\left(\mu\left(\frac{\partial w}{\partial y}+\frac{\partial v}{\partial z}\right)\right)}{\partial y}+\frac{\partial\left(2 \mu \frac{\partial w}{\partial z}-p\right)}{\partial z}=\rho g, \\
& \frac{\partial u}{\partial x}+\frac{\partial v}{\partial y}+\frac{\partial w}{\partial z}=0,
\end{aligned}
$$

where $(u, v, w)^{T}$ denotes the velocity, $p$ the pressure, $\rho$ the density of ice, and $g$ the gravitational acceleration. The effective viscosity $\mu$ is defined by Glen's flow (Nye, 1957) law as

$\mu=\frac{1}{2} A^{-\frac{1}{n}}\left(\frac{1}{2} \dot{\varepsilon}: \dot{\varepsilon}\right)^{\frac{1-n}{2 n}}$,

where $n$ is the power-law exponent ( $n=3$ is generally assumed for modeling ice), $A$ is the temperature-dependent deformation rate factor, and $\dot{\varepsilon}$ is the strain-rate tensor defined as $\dot{\varepsilon}=\frac{1}{2}\left(B+B^{T}\right)$ with $B=(\nabla u, \nabla v, \nabla w)^{T}$. In the isothermal case, $A$ is taken as a spatially and temporally uniform constant.

If the top surface of the ice sheet is allowed to evolve in time, then a prognostic equation describing the evolution of that free surface is included. The ice-sheet domain $\Omega_{t}$ at each time $t \in\left[0, t_{\max }\right]$ can be defined as

$$
\begin{aligned}
\Omega_{t}= & \{(x, y, z) \mid b(x, y) \leq z \leq s(x, y, t) \\
& \text { for } \left.(x, y) \in \Omega_{\mathrm{H}}\right\},
\end{aligned}
$$

where $\Omega_{\mathrm{H}}$ denotes the horizontal extent of the ice sheet, $s(x, y, t)$ defines the top surface elevation, and $b(x, y)$ defines the fixed bottom surface of the ice sheet. We denote the top surface as $\Gamma_{\mathrm{s}}$ and the bottom surface as $\Gamma_{\mathrm{b}}$. The motion of the free surface is governed by the kinematic relation

$$
\frac{\partial s}{\partial t}+u \frac{\partial s}{\partial x}+v \frac{\partial s}{\partial y}-w=a, \quad(x, y) \in \Omega_{\mathrm{H}},
$$

on the top surface of the ice sheet $\Gamma_{\mathrm{s}}$, where $a$ represents the surface mass balance (accumulation minus ablation). Because the bed of the ice sheet is assumed to be fixed and the melting/refreezing rate is neglected, we obtain a similar kinematic relation,

$$
u \frac{\partial b}{\partial x}+v \frac{\partial b}{\partial y}-w=0, \quad(x, y) \in \Omega_{\mathrm{H}},
$$

on the bottom surface $\Gamma_{\mathrm{b}}$.

\subsection{Boundary conditions}

At the top surface $\Gamma_{\mathrm{s}}$ of the ice sheet, we impose a stress free boundary condition:

$$
\begin{gathered}
\frac{1}{r_{\mathrm{s}}}\left[-\frac{\partial s}{\partial x}\left(2 \mu \frac{\partial u}{\partial x}-p\right)-\frac{\partial s}{\partial y} \mu\left(\frac{\partial u}{\partial y}+\frac{\partial v}{\partial x}\right)+\mu\left(\frac{\partial u}{\partial z}+\frac{\partial w}{\partial x}\right)\right]=0, \\
\frac{1}{r_{\mathrm{s}}}\left[-\frac{\partial s}{\partial x} \mu\left(\frac{\partial u}{\partial y}+\frac{\partial v}{\partial x}\right)-\frac{\partial s}{\partial y}\left(2 \mu \frac{\partial v}{\partial y}-p\right)+\mu\left(\frac{\partial v}{\partial z}+\frac{\partial w}{\partial y}\right)\right]=0, \\
\frac{1}{r_{\mathrm{s}}}\left[-\frac{\partial s}{\partial x} \mu\left(\frac{\partial u}{\partial z}+\frac{\partial w}{\partial x}\right)-\frac{\partial s}{\partial y} \mu\left(\frac{\partial w}{\partial y}+\frac{\partial v}{\partial z}\right)+\left(2 \mu \frac{\partial w}{\partial z}-p\right)\right]=0,
\end{gathered}
$$



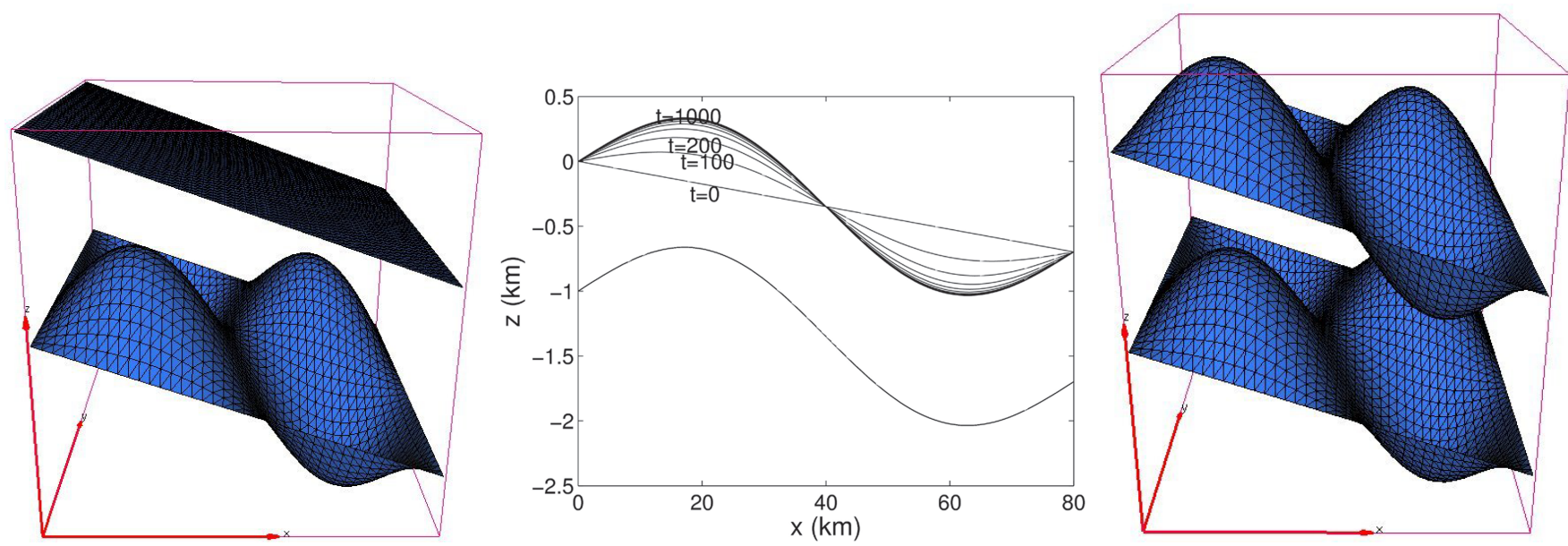

Fig. 1. Illustration of the ice-sheet geometry (the top and bottom surfaces) at the time $t=0$ (left) and at the time $t=1000 \mathrm{yr}$ (right). The middle figure is the $x$-direction profiles taken at $y=L / 4$ of the ice-sheet top and bottom surfaces at 100 year time intervals from $t=0$ to $1000 \mathrm{yr}$.

and where $r_{s}=\sqrt{1+\left(\frac{\partial s}{\partial x}\right)^{2}+\left(\frac{\partial s}{\partial y}\right)^{2}}$

The bottom bedrock surface $\Gamma_{\mathrm{b}}$ of the ice sheet can be decomposed into two parts: $\Gamma_{\mathrm{b}, \mathrm{fix}}$, at which the ice sheet is fixed to the bottom bedrock, and $\Gamma_{\mathrm{b} \text {,sld }}$, at which it is allowed to slip. We apply the zero velocity (no-slip and no-penetration) boundary condition

$u=v=w=0$

on the fixed part of the basal boundary $\Gamma_{\mathrm{b}, \mathrm{fix}}$ and the Rayleigh friction boundary condition

$$
\begin{aligned}
& \frac{1}{r_{b}}\left[-\frac{\partial b}{\partial x}\left(2 \mu \frac{\partial u}{\partial x}-p\right)-\frac{\partial b}{\partial y} \mu\left(\frac{\partial u}{\partial y}+\frac{\partial v}{\partial x}\right)+\mu\left(\frac{\partial u}{\partial z}+\frac{\partial w}{\partial x}\right)\right]+\frac{1}{r_{\mathrm{b}}} \frac{\partial b}{\partial x} f=-\beta^{2} u, \\
& \frac{1}{r_{\mathrm{b}}}\left[-\frac{\partial b}{\partial x} \mu\left(\frac{\partial u}{\partial y}+\frac{\partial v}{\partial x}\right)-\frac{\partial b}{\partial y}\left(2 \mu \frac{\partial v}{\partial y}-p\right)+\mu\left(\frac{\partial v}{\partial z}+\frac{\partial w}{\partial y}\right)\right]+\frac{1}{r_{\mathrm{b}}} \frac{\partial b}{\partial y} f=-\beta^{2} v, \\
& u \frac{\partial b}{\partial x}+v \frac{\partial b}{\partial y}-w=0
\end{aligned}
$$

on the sliding part $\Gamma_{\mathrm{b}, \mathrm{sld}}$, where $r_{\mathrm{s}}=\sqrt{1+\left(\frac{\partial b}{\partial x}\right)^{2}+\left(\frac{\partial b}{\partial y}\right)^{2}}$,

$$
\begin{aligned}
f= & \frac{1}{r_{b}^{2}}\left[-\frac{\partial b}{\partial x}\left(-\frac{\partial b}{\partial x}\left(2 \mu \frac{\partial u}{\partial x}-p\right)-\frac{\partial b}{\partial y} \mu\left(\frac{\partial u}{\partial y}+\frac{\partial v}{\partial x}\right)+\mu\left(\frac{\partial u}{\partial z}+\frac{\partial w}{\partial x}\right)\right)\right. \\
& -\frac{\partial b}{\partial y}\left(-\frac{\partial b}{\partial x} \mu\left(\frac{\partial u}{\partial y}+\frac{\partial v}{\partial x}\right)-\frac{\partial b}{\partial y}\left(2 \mu \frac{\partial v}{\partial y}-p\right)+\mu\left(\frac{\partial v}{\partial z}+\frac{\partial w}{\partial y}\right)\right) \\
& \left.+\left(-\frac{\partial b}{\partial x} \mu\left(\frac{\partial u}{\partial z}+\frac{\partial w}{\partial x}\right)-\frac{\partial b}{\partial y}\left(\frac{\partial v}{\partial z}+\frac{\partial w}{\partial y}\right)+\mu\left(2 \mu \frac{\partial w}{\partial z}-p\right)\right)\right]
\end{aligned}
$$

is the normal stress, and the parameter $\beta^{2}$ denotes a given, positive sliding coefficient.

Note that the zero velocity boundary condition Eq. (12) automatically implies the kinematic condition Eq. (8), whereas the latter condition is included as the third equation of the sliding condition Eqs. (13)-(15). If $\Gamma_{\mathrm{b}, \mathrm{fix}}=\Gamma_{\mathrm{b}}$, we have a pure zero velocity boundary condition on the bedrock surface; if $\Gamma_{\mathrm{b}, \text { sld }}=\Gamma_{\mathrm{b}}$, we have a pure sliding boundary condition; otherwise, we have a mixed boundary condition.
We also assume that $b(x, y) \neq s(x, y, t)$ along the boundary of $\Omega_{\mathrm{H}}$, so that the ice sheet has a lateral boundary $\Gamma_{\ell}$ with some appropriate boundary conditions imposed there; for example, a periodic boundary condition or a zero velocity boundary condition could be applied there.

\section{Manufactured analytic solutions}

\subsection{A general form of the analytic solution}

Following the work of Sargent and Fastook (2010), we start from the kinematic boundary conditions Eqs. (7) and (8) on the top and bottom surfaces, respectively, and set the vertical velocity $w$ by linearly interpolating $u$ and $v$ from the top to bottom bedrock surfaces as follows:

$$
\begin{aligned}
w(x, y, z, t) & =u(x, y, z, t)\left(\frac{\partial b}{\partial x} \frac{s-z}{s-b}+\frac{\partial s}{\partial x} \frac{z-b}{s-b}\right) \\
& +v(x, y, z, t)\left(\frac{\partial b}{\partial y} \frac{s-z}{s-b}+\frac{\partial s}{\partial y} \frac{z-b}{s-b}\right) \\
& +\left(\frac{\partial s}{\partial t}-a\right) \frac{z-b}{s-b} .
\end{aligned}
$$

Differentiating Eq. (17) with respect to $z$ then gives

$$
\begin{aligned}
\frac{\partial w}{\partial z} & =\frac{\partial u}{\partial z}\left(\frac{\partial b}{\partial x} \frac{s-z}{s-b}+\frac{\partial s}{\partial x} \frac{z-b}{s-b}\right) \\
& +u \frac{\frac{\partial s}{\partial x}-\frac{\partial b}{\partial x}}{s-b}+\frac{\partial v}{\partial z}\left(\frac{\partial b}{\partial y} \frac{s-z}{s-b}+\frac{\partial s}{\partial y} \frac{z-b}{s-b}\right)+v \frac{\frac{\partial s}{\partial y}-\frac{\partial b}{\partial y}}{s-b} \\
& +\frac{1}{s-b}\left(\frac{\partial s}{\partial t}-a\right) .
\end{aligned}
$$

Now substituting Eq. (18) into the incompressibility equation Eq. (4), we obtain a first-order quasi-linear partial differential equation with three independent variables, $x, y, z$, and 

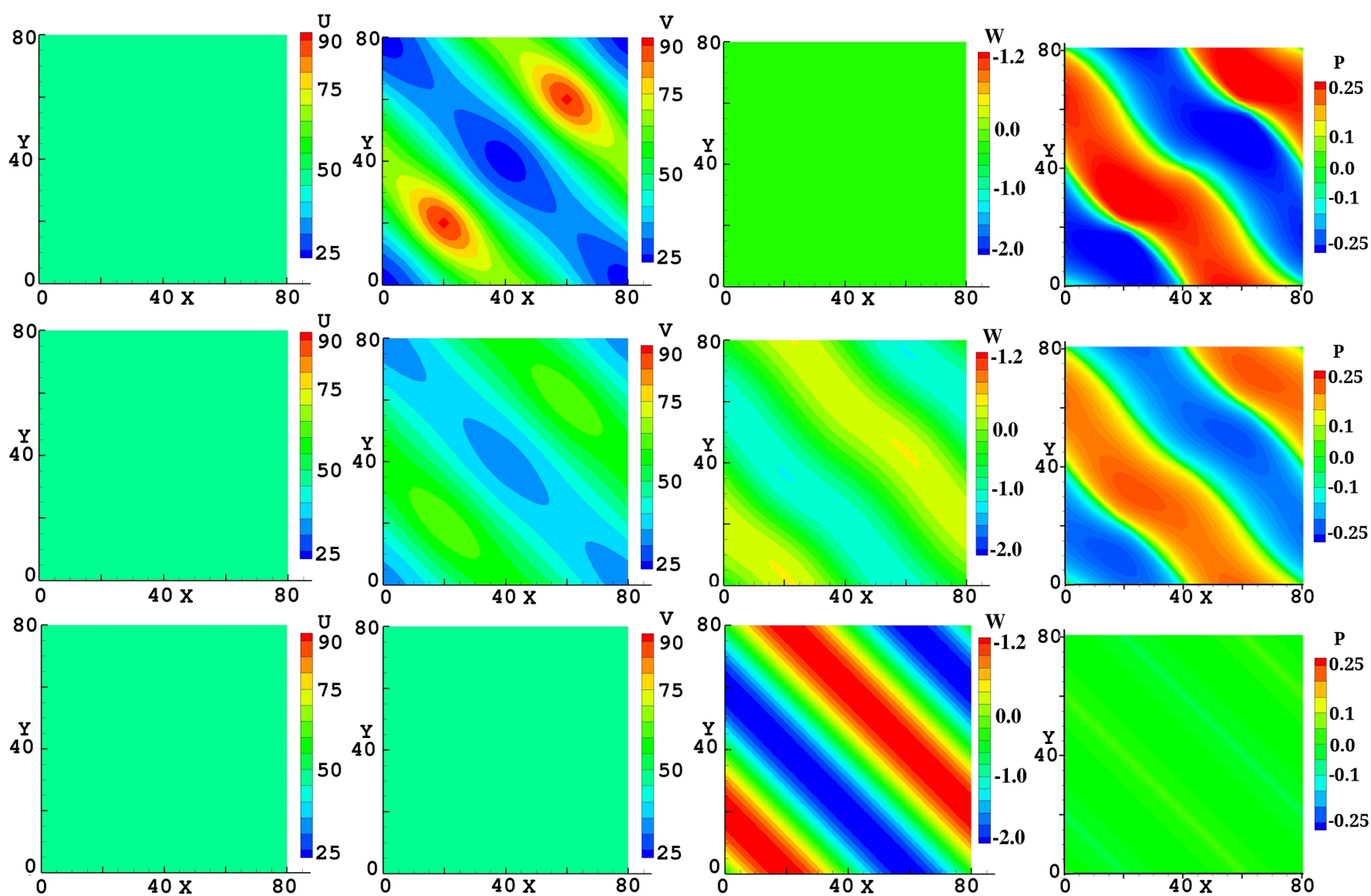

Fig. 2. Analytical solution at the top surface of the ice sheet. From left to right: the velocity components $u, v$, and $w$ (m a ${ }^{-1}$ ) and the pressure $p\left(10^{5} \mathrm{~Pa}\right)$. From top to bottom: $t=0,100$, and $1000 \mathrm{yr}$.

two dependent variables, $u$ and $v$ :

$$
\begin{aligned}
& \frac{\partial u}{\partial x}+\frac{\partial u}{\partial z}\left(\frac{\partial b}{\partial x}\right.\left.\frac{s-z}{s-b}+\frac{\partial s}{\partial x} \frac{z-b}{s-b}\right)+u \frac{\frac{\partial s}{\partial x}-\frac{\partial b}{\partial x}}{s-b} \\
&+\frac{\partial v}{\partial y}+\frac{\partial v}{\partial z}\left(\frac{\partial b}{\partial y} \frac{s-z}{s-b}+\frac{\partial s}{\partial y} \frac{z-b}{s-b}\right) \\
&+v \frac{\frac{\partial s}{\partial y}-\frac{\partial b}{\partial y}}{s-b}+\frac{1}{s-b}\left(\frac{\partial s}{\partial t}-a\right)=0 .
\end{aligned}
$$

Let us choose the velocity $u(x, y, z, t)$ to be of a form similar to the manufactured 2-D analytical solution in Sargent and Fastook (2010),

$u(x, y, z, t)=c_{1}(s-b)^{\gamma_{1}}\left[1-\left(\frac{s-z}{s-b}\right)^{\lambda_{1}}\right]+c_{\mathrm{b} 1} \frac{1}{s-b}$,

where $\gamma_{1}, \lambda_{1}, c_{1}$, and $c_{\mathrm{b} 1}$ are some parameters. If we define the scaled ice depth

$d(x, y, z, t)=\frac{s-z}{s-b}$,

and the ice thickness

$h(x, y, t)=s-b$, then Eq. (20) can be expressed as

$u(x, y, z, t)=c_{1} h^{\gamma_{1}}\left(1-d^{\lambda_{1}}\right)+c_{\mathrm{b} 1} \frac{1}{h}$.

Note that $s, b$, and $h$ all depend on $x$ and $y$, but are independent of $z$.

It is then easy to verify that the derivatives of $u(x, y, z, t)$ are given by

$$
\begin{aligned}
\frac{\partial u}{\partial x} & =c_{1} \gamma_{1} h^{\gamma_{1}-1} \frac{\partial h}{\partial x}\left(1-d^{\lambda_{1}}\right)-c_{1} \lambda_{1} h^{\gamma_{1}} d^{\lambda_{1}-1} \frac{\partial d}{\partial x}-c_{\mathrm{b} 1} \frac{1}{h^{2}} \frac{\partial h}{\partial x} \\
\frac{\partial u}{\partial z} & =c_{1} \lambda_{1} h^{\gamma_{1}-1} d^{\lambda_{1}-1} .
\end{aligned}
$$

Substituting Eqs. (24)-(25) into Eq. (19), we obtain a new first-order quasi-linear partial differential equation with two independent variables, $y, z$, and one dependent variable $v$ :

$$
\begin{aligned}
\frac{\partial v}{\partial y}+\frac{\partial v}{\partial z} & \left(\frac{\partial b}{\partial y} \frac{s-z}{s-b}+\frac{\partial s}{\partial y} \frac{z-b}{s-b}\right)+v \frac{\frac{\partial h}{\partial y}}{h} \\
& +c_{1}\left(1+\gamma_{1}\right) \frac{\partial h}{\partial x} h^{\gamma_{1}-1}\left(1-d^{\lambda_{1}}\right)+\frac{1}{h}\left(\frac{\partial s}{\partial t}-a\right)=0 .
\end{aligned}
$$

Note that the variable $v$ in general depends on $x, y, z$, and $t$; however, the partial differential Eq. (26) now only involves 

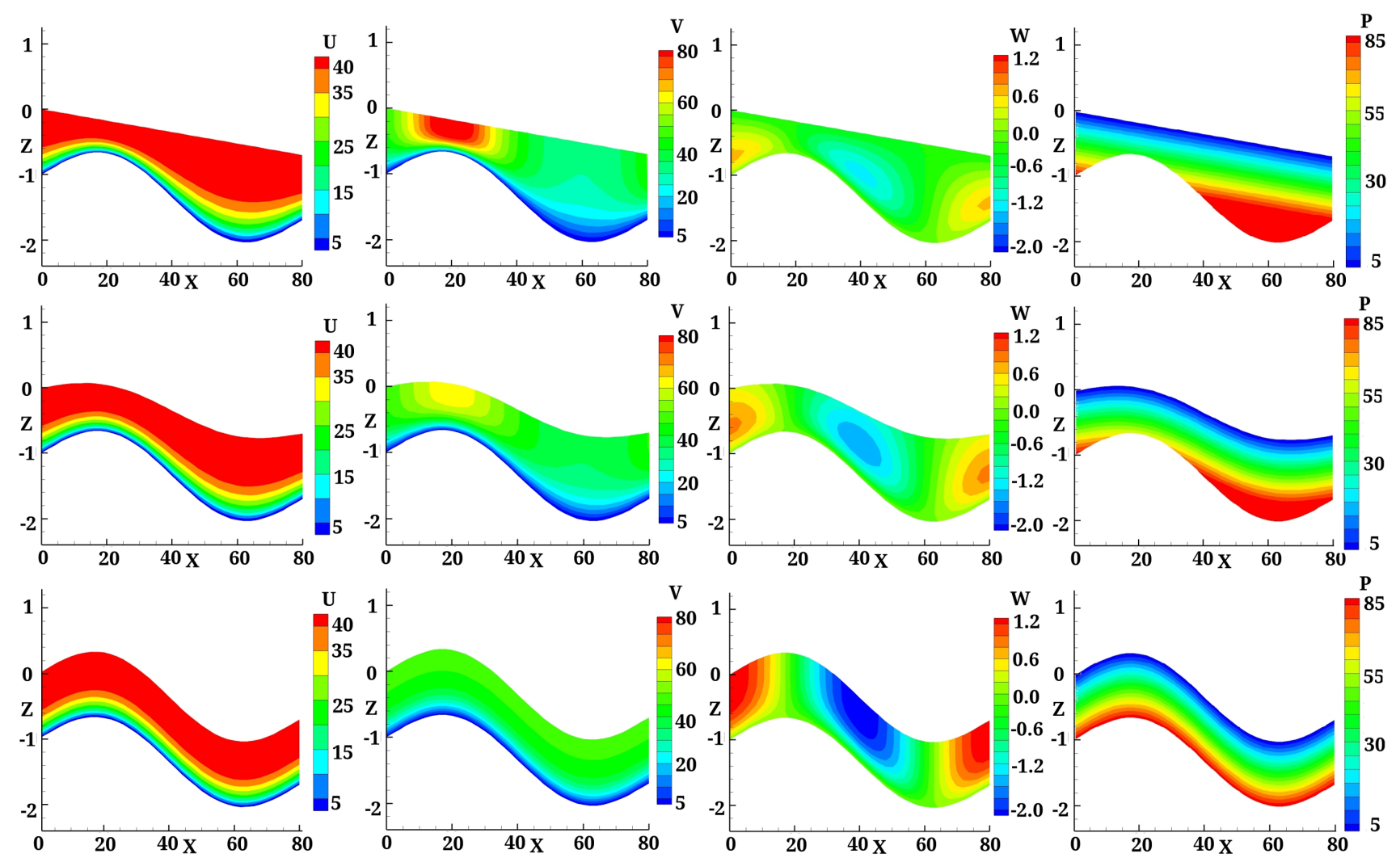

Fig. 3. Analytical solution along the cross section $y=L / 4$ of the ice sheet. From left to right: the velocity components $u, v$, and $w$ (m a ${ }^{-1}$ ) and the pressure $p\left(10^{5} \mathrm{~Pa}\right)$. From top to bottom: $t=0,100$, and $1000 \mathrm{yr}$.

partial differentials of $y$ and $z$ at a fixed time $t$. Thus, in the following deductions, the total differential $\mathrm{d}(\cdot)$ is on variables $y$ and $z$ only, e.g., $\mathrm{d}(g(f(y, z)))=\frac{\mathrm{d} g}{\mathrm{~d} f}\left(\frac{\partial f}{\partial y} \mathrm{~d} y+\frac{\partial f}{\partial z} \mathrm{~d} z\right)$.

In the work of Sargent and Fastook (2010), the first-order partial differential Eq. (26) was solved incorrectly; it resulted in a wrong solution for $v$ and consequently the wrong general formula for the analytical solution and the specific solution under a special geometry. We present the correct solution process in the following.

The characteristic equation of Eq. (26) can be found as

$$
\begin{aligned}
\frac{\mathrm{d} y}{1} & =\frac{\mathrm{d} z}{\frac{\partial b}{\partial y} \frac{s-z}{s-b}+\frac{\partial s}{\partial y} \frac{z-b}{s-b}} \\
& =-\frac{\mathrm{d} v}{v \frac{\frac{\partial h}{\partial y}}{h}+c_{1}\left(1+\gamma_{1}\right) \frac{\partial h}{\partial x} h^{\gamma_{1}-1}\left(1-d^{\lambda_{1}}\right)+\frac{1}{h}\left(\frac{\partial s}{\partial t}-a\right)} .
\end{aligned}
$$

Note that the first-order partial differential Eq. (26) does not need initial conditions. To solve it we first need to find two independent integrable identities that, when integrated, provide equations such as

$\left\{\begin{array}{l}\phi(y, z, v)=C_{1}, \\ \psi(y, z, v)=C_{2},\end{array}\right.$ where $C_{1}$ and $C_{2}$ are two underdetermined constants. The solution of Eq. (26) then can be written as

$\theta(\phi, \psi)=0$,

where $\theta$ is an arbitrary smooth function of $\phi$ and $\psi$.

The first integral can be deduced from

$$
\frac{\mathrm{d} y}{1}=\frac{\mathrm{d} z}{\frac{\partial b}{\partial y} \frac{s-z}{s-b}+\frac{\partial s}{\partial y} \frac{z-b}{s-b}},
$$

from which we then have

$\mathrm{d}\left(\frac{z-b}{s-b}\right)=0$,

implying that

$\frac{z-b}{s-b}=C_{1}$.

Thus we take

$\phi=\frac{z-b}{s-b}$.

The second integral can be deduced from

$\frac{\mathrm{d} y}{1}=-\frac{\mathrm{d} v}{v \frac{\partial h}{\frac{\partial y}{h}}+c_{1}\left(1+\gamma_{1}\right) \frac{\partial h}{\partial x} h^{\gamma_{1}-1}\left(1-d^{\lambda_{1}}\right)+\frac{1}{h}\left(\frac{\partial s}{\partial t}-a\right)}$, 

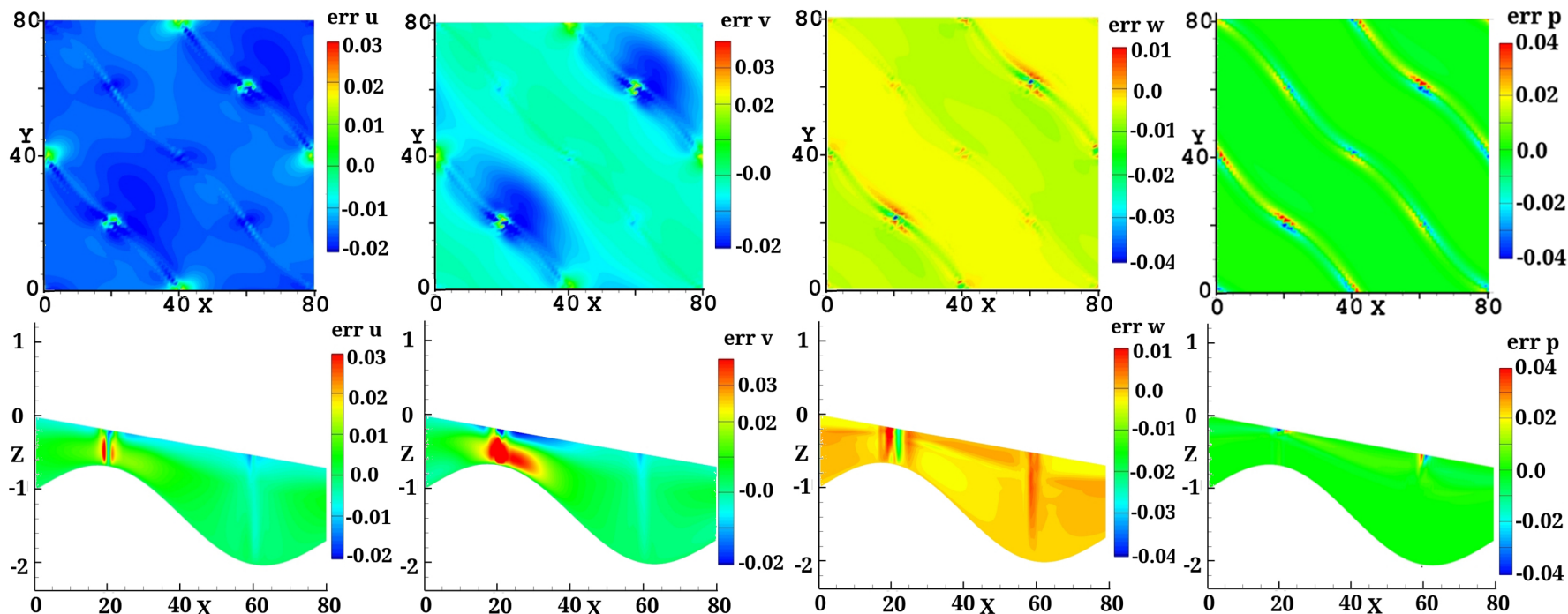

Fig. 4. Distribution of errors of the numerical simulations using the grid of resolution $160 \times 160 \times 40$ at time $t=0$. From left to right: the velocity components $u, v$ and $w\left(\mathrm{~m} \mathrm{a}^{-1}\right)$ and the pressure $p\left(10^{5} \mathrm{~Pa}\right)$. Top row: on the top surface, bottom row: along the cross section $y=L / 4$.

from which we then have

$\mathrm{d}(h v)=-c_{1}\left(1+\gamma_{1}\right)\left[1-\left(\frac{s-z}{s-b}\right)^{\lambda_{1}}\right] \frac{\partial h}{\partial x} h^{\gamma_{1}} \mathrm{~d} y-\left(\frac{\partial s}{\partial t}-a\right) \mathrm{d} y$

Note that $\mathrm{d}\left(\frac{z-b}{s-b}\right)=0$, in which case the integration of Eq. (35) gives

$h v=-c_{1}\left(1+\gamma_{1}\right)\left[1-\left(\frac{s-z}{s-b}\right)^{\lambda_{1}}\right] \int \frac{\partial h}{\partial x} h^{\gamma_{1}} \mathrm{~d} y-\int\left(\frac{\partial s}{\partial t}-a\right) \mathrm{d} y+C_{2}$.

Thus we set

$$
\begin{gathered}
\psi=v(s-b)+c_{1}\left(1+\gamma_{1}\right)\left[1-\left(\frac{s-z}{s-b}\right)^{\lambda_{1}}\right] \int\left(\frac{\partial s}{\partial x}-\frac{\partial b}{\partial x}\right) \\
(s-b)^{\gamma_{1}} \mathrm{~d} y+\int\left(\frac{\partial s}{\partial t}-a\right) \mathrm{d} y .
\end{gathered}
$$

The combination of Eqs. (29), (33), and (37) implies that the general solution of Eq. (26) can be written as

$$
\begin{gathered}
\theta\left(\frac{z-b}{s-b}, v(s-b)+c_{1}\left(1+\gamma_{1}\right)\left[1-\left(\frac{s-z}{s-b}\right)^{\lambda_{1}}\right] \int\left(\frac{\partial s}{\partial x}-\frac{\partial b}{\partial x}\right)(s-b)^{\gamma_{1}} \mathrm{~d} y\right. \\
\left.+\int\left(\frac{\partial s}{\partial t}-a\right) \mathrm{d} y\right)=0
\end{gathered}
$$

for some smooth function $\theta(\cdot, \cdot)$.

To better represent the relationship between the velocity $v$ and the depth $d$, we choose the function $\theta$ as

$\theta(\phi, \psi)=\psi-c_{2}\left[1-(1-\phi)^{\lambda_{2}}\right]+c_{\mathrm{b} 2}$,

where $\lambda_{2}, c_{2}$, and $c_{\mathrm{b} 2}$ are again some parameters. It is then easy to verify that $v$ can be written in the form

$$
\begin{aligned}
& v(x, y, z, t)=\frac{c_{2}}{s-b}\left[1-\left(\frac{s-z}{s-b}\right)^{\lambda_{2}}\right]+c_{b 2} \frac{1}{s-b}-\frac{c_{1}}{s-b}\left(1+\gamma_{1}\right)\left[1-\left(\frac{s-z}{s-b}\right)^{\lambda_{1}}\right] \\
& \cdot \int\left(\frac{\partial s}{\partial x}-\frac{\partial b}{\partial x}\right)(s-b)^{\gamma_{1}} \mathrm{~d} y-\frac{1}{s-b} \int\left(\frac{\partial s}{\partial t}-a\right) \mathrm{d} y,
\end{aligned}
$$

which is different from the one presented in Sargent and Fastook (2010).

By combining Eqs. (17), (23), and (40), we finally obtain the velocity solution derived from the kinematic boundary condition and the mass conservation equation as follows:

$$
\begin{aligned}
u(x, y, z, t) & =c_{1}(s-b)^{\gamma_{1}}\left[1-\left(\frac{s-z}{s-b}\right)^{\lambda_{1}}\right]+c_{b 1} \frac{1}{s-b}, \\
v(x, y, z, t)= & \frac{c_{2}}{s-b}\left[1-\left(\frac{s-z}{s-b}\right)^{\lambda_{2}}\right]+c_{b 2} \frac{1}{s-b}-\frac{c_{1}}{s-b}\left(1+\gamma_{1}\right)\left[1-\left(\frac{s-z}{s-b}\right)^{\lambda_{1}}\right] \\
& \cdot \int\left(\frac{\partial s}{\partial x}-\frac{\partial b}{\partial x}\right)(s-b)^{\gamma_{1}} \mathrm{~d} y-\frac{1}{s-b} \int\left(\frac{\partial s}{\partial t}-a\right) \mathrm{d} y, \\
w(x, y, z, t) & =u(x, y, z, t)\left(\frac{\partial b}{\partial x} \frac{s-z}{s-b}+\frac{\partial s}{\partial x} \frac{z-b}{s-b}\right)+v(x, y, z, t)\left(\frac{\partial b}{\partial y} \frac{s-z}{s-b}+\frac{\partial s}{\partial y} \frac{z-b}{s-b}\right) \\
& +\left(\frac{\partial s}{\partial t}-a\right) \frac{z-b}{s-b} .
\end{aligned}
$$

We choose the pressure solution to be that from the firstorder ice-sheet model (Pattyn, 2003):

$p(x, y, z, t)=-2 \mu \frac{\partial u}{\partial x}-2 \mu \frac{\partial v}{\partial y}+\rho g(s-z)$.

Equation (44) is derived from the Stokes momentum Eqs. (1)-(3) and the stress-free boundary conditions Eqs. (9)-(11) on the top surface through a first-order approximation. Consequently, the above manufactured analytic velocity and pressure solutions in Eqs. (41)-(44) do not satisfy exactly the momentum equations and the top surface boundary equations. In order to maintain the equalities of these equations, some additional compensation terms need be added to the right-hand sides of the Eqs. (1)-(3) and (9)(11); these terms can be easily obtained by way of substituting the above constructed analytical solution in Eqs. (41)(43) and Eq. (44) into the left-hand sides of Eqs. (1)-(3) and Eqs. (9)-(11). The explicit formulas for calculating these 

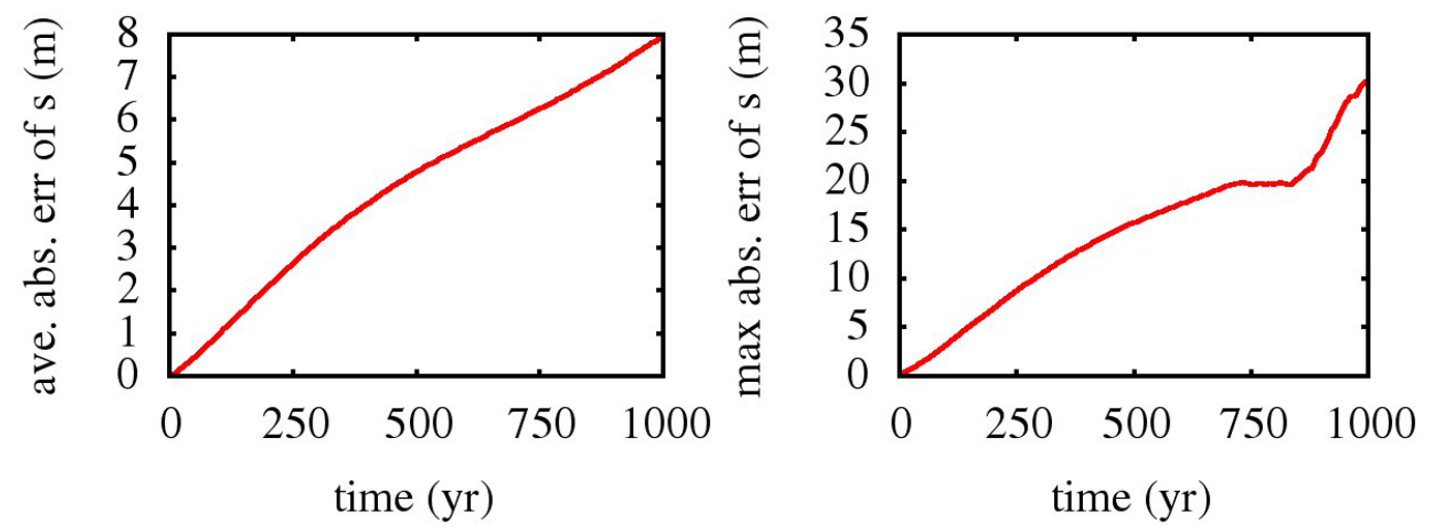

Fig. 5. Plot of the average (left) and the maximum (right) absolute errors of the simulated top surface elevation $s$ (m) at each of the time steps for the time-dependent ice-sheet flow experiment using the grid of resolution $80 \times 80 \times 20$.

compensation terms for the manufactured solution under the specific geometry presented in the next section are given in the supplemental materials. We further note that if a sliding boundary condition is imposed on the (full or partial) bottom bedrock surface, then the right-hand sides of Eqs. (13) and (14) also need to be slightly revised as in the above process.

\subsection{A manufactured solution under a specific geometry for time-dependent ice flow}

Given the surface and bed elevation (i.e., the geometry of an ice sheet), a specific manufactured solution can be produced using the above procedure from Eqs. (41)-(44). Our goal here is to present the ice-sheet modeling community a correct, practically useful and simple sample solution to verify the computational 3-D Stokes ice-sheet models. To simplify the formulation, we introduce some scaling parameters as follows: $L$ is the horizontal length scale (span) of the ice sheet, $Z$ is the vertical length scale, $\delta=L / Z$ is the aspect ratio, $U=A L(2 \rho g Z)^{n}$ is the horizontal velocity scale, $W=U Z / L$ is the vertical velocity scale, and $T=Z / W$ is the time scale. Note that we do not use non-dimensionalized variables or equations; the scaling parameters are used only for a clearer representation of the derived solutions.

The geometric setting for the the ISMIP-HOM benchmark experiment A (Pattyn et al., 2008) is used here; a parallelsided slab of ice with a mean thickness of $Z$ and length $L$ lies on a bed with a mean slope of $\alpha=0.5^{\circ}$. The top surface of the ice sheet at the initial time $t=0$ is given by

$s(x, y, 0)=s_{0}(x, y)=-x \tan (\alpha)$.

The fixed basal topography is defined as a series of $Z / 2$ amplitude sinusoidal oscillations about the mean bed elevation:

$b(x, y)=s_{0}(x, y)+\eta(x, y)-Z$

with

$\eta(x, y)=\frac{Z}{2} \sin \left(\frac{2 \pi x}{L}\right) \sin \left(\frac{2 \pi y}{L}\right)$.
Evolving over time, the top surface of the ice sheet slowly changes from flat with a uniform slope to sinusoidal in shape:

$s(x, y, t)=s_{0}(x, y)+\eta(x, y) \xi(t)$

with

$\xi(t)=1-e^{-c_{t} t}$,

where $c_{\mathrm{t}}$ is a parameter that controls the rate of ice-thickness change.

We assume a periodic surface mass balance given by

$a(x, y, t)=c_{t} \eta(x, y) e^{-c_{t} t}$.

Then, it is easy to verify that

$\frac{\partial s}{\partial t}-a=0$

and thus

$-\frac{1}{s-b} \int_{\frac{L}{4}}^{y}\left(\frac{\partial s}{\partial t}-a\right) \mathrm{d} y=0$.

Based on the above known functions $s, b$, and $a$ and the parameters $\gamma_{1}, \lambda_{1}, \lambda_{2}, c_{1}, c_{2}, c_{\mathrm{b} 1}, c_{\mathrm{b} 2}$, and $c_{\mathrm{t}}$, we construct the velocity solution $(u, v, w)$ and the pressure solution $p$ using Eqs. (41)-(44) for the time-dependent, isothermal, 3D Stokes ice-sheet flow model. Note that this manufactured solution is obviously doubly periodic in velocity so that a periodic boundary condition on the lateral boundary $\Gamma_{1}\left(\Gamma_{1} \neq\right.$ $\emptyset)$ is satisfied.

To further simplify the computation of the integral in Eq. (42), we set $\gamma_{1}=0$ and $\lambda_{1}=4$. Then the velocity component $v$ defined in Eq. (42) simplifies to

$$
\begin{aligned}
& v(x, y, z, t)=\frac{c_{2}}{s-b}\left[1-\left(\frac{s-z}{s-b}\right)^{\lambda_{2}}\right]+c_{b 2} \frac{1}{s-b} \\
& -\frac{1}{2} \frac{c_{1}}{s-b}\left[1-\left(\frac{s-z}{s-b}\right)^{4}\right] Z \cos \left(\frac{2 \pi x}{L}\right) \cos \left(\frac{2 \pi y}{L}\right) e^{-c_{1} t},
\end{aligned}
$$



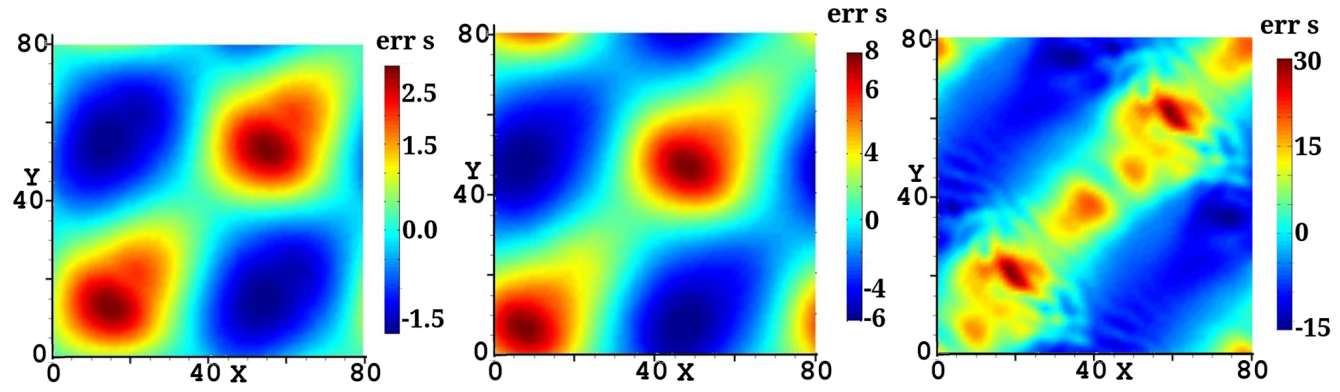

Fig. 6. Distribution of the errors of the simulated top surface elevation $s(\mathrm{~m})$ for the time-dependent ice-sheet flow experiment using the grid of resolution $80 \times 80 \times 20$. From left to right: at 100,300 , and $1000 \mathrm{yr}$.

which is much easier to calculate.

Additionally, we also let $c_{\mathrm{b} 1}=c_{\mathrm{b} 2}=0$; then, the velocity solution Eqs. (41)-(43) can be finally simplified as

$$
\begin{aligned}
& u(x, y, z, t)=c_{1}\left[1-\left(\frac{s-z}{s-b}\right)^{4}\right], \\
& v(x, y, z, t)=\frac{c_{2}}{s-b}\left[1-\left(\frac{s-z}{s-b}\right)^{\lambda_{2}}\right] \\
& -\frac{1}{2} \frac{c_{1}}{s-b}\left[1-\left(\frac{s-z}{s-b}\right)^{4}\right] Z \cos \left(\frac{2 \pi x}{L}\right) \cos \left(\frac{2 \pi y}{L}\right) e^{-c_{\mathrm{t}} t} \text {, } \\
& w(x, y, z, t)=u(x, y, z, t)\left(\frac{\partial b}{\partial x} \frac{s-z}{s-b}+\frac{\partial s}{\partial x} \frac{z-b}{s-b}\right) \\
& +v(x, y, z, t)\left(\frac{\partial b}{\partial y} \frac{s-z}{s-b}+\frac{\partial s}{\partial y} \frac{z-b}{s-b}\right) .
\end{aligned}
$$

The solution $(u, v, w)$ defined by Eqs. (54)-(56) satisfies a pure zero-velocity boundary condition on the whole bedrock surface $\Gamma_{\mathrm{b}, \mathrm{fix}}=\Gamma_{\mathrm{b}}$. The parameters $c_{1}, c_{2}$, and $c_{\mathrm{t}}$ can be used to control the velocity falling within a reasonable range. In general, the larger $c_{1}$ and $c_{2}$ are, the larger the magnitude of the velocity of the ice sheet and its variation are. The bigger $c_{\mathrm{t}}$ is, the shorter the time for the ice sheet to reach the steady state is.

Note that the velocity solution $(u, v, w)$ of the (slightly modified) Stokes equation at the initial time $t=0$ could easily be generated by setting the function $\xi(t)=0$ in the analytical time-dependent solutions. Similarly, the final geometrically stable ice-sheet configuration (i.e., a steady state with $\left.\frac{\mathrm{d} s}{\mathrm{~d} t}=0\right)$ will have a top surface with the elevation function given by $s_{0}(x, y)+\eta(x, y)$, generated by setting the function $\xi(t)=1$ in the analytical time-dependent solution. We also would like to point out that the values of the parameters chosen in this paper for the specific example are different from those used in Sargent and Fastook (2010) and give us a much simpler specific solution and compensatory terms based on the correct general solution formula.

\section{Numerical verification of the Stokes ice-sheet model of Leng et al. (2012)}

We use the above manufactured analytic solutions to verify the parallel, high-order accurate, finite element, nonlinear Stokes ice-sheet model in Leng et al. (2012). The model uses tetrahedral elements that are produced by first extruding a 2$\mathrm{D}$, triangular mesh in the $x, y$-planes along the $z$-direction, after which each vertical prism is decomposed into three tetrahedral elements. The stable Taylor-Hood (P2-P1) finite element pair is used. A pure zero-velocity boundary condition is imposed on the whole bedrock surface $\Gamma_{\mathrm{b}}$ (no sliding region). The free-surface boundary condition with compensatory terms is imposed on the top surface $\Gamma_{\mathrm{s}}$ and periodic boundary conditions are imposed on the lateral boundaries $\Gamma_{1}$.

\subsection{Description of the manufactured analytical solution}

We set $\gamma_{1}=0, \lambda_{1}=4, c_{\mathrm{b} 1}=0$, and $c_{\mathrm{b} 2}=0$, as discussed in the previous section; the other parameters used in the construction of the manufactured solution are chosen as follows: $\lambda_{2}=4, c_{1}=10^{-9} U, c_{2}=10^{-9} U$ and $c_{\mathrm{t}}=10^{-8} / T$. The horizontal length scale of the ice sheet is set to be $L=80$ $\mathrm{km}$ and the vertical length scale $Z=1 \mathrm{~km}$. For the parameters related to physical properties of the ice sheet, we set $A=10^{-16}\left(\mathrm{~Pa}^{-n} \mathrm{a}^{-1}\right)$ and $n=3$. Note that the $\mathrm{C}$ code for calculating the compensation terms of this particular example can be found in the supplemental materials.

After $t=1000 \mathrm{yr}$ of evolution, the manufactured solution for the ice-sheet surface $s(x, y, t)$, as defined in Eq. (48), will be close to steady state such that no significant changes in the free surface occur. Initially, the top surface is flat with a uniform slope (Fig. 1, left). When the final steady state is essentially reached (at $1000 \mathrm{yr}$ ), the surface takes on the sinusoidal shape of the bed topography and the ice thickness is everywhere uniform and equal (Fig. 1, right). Figure 1 (middle) illustrates the evolution of the ice surface at 100-yr time intervals along a selected profile line.

Figure 2 presents the three velocity components and the pressure of the analytical solution defined by Eqs. (54)-(56) 


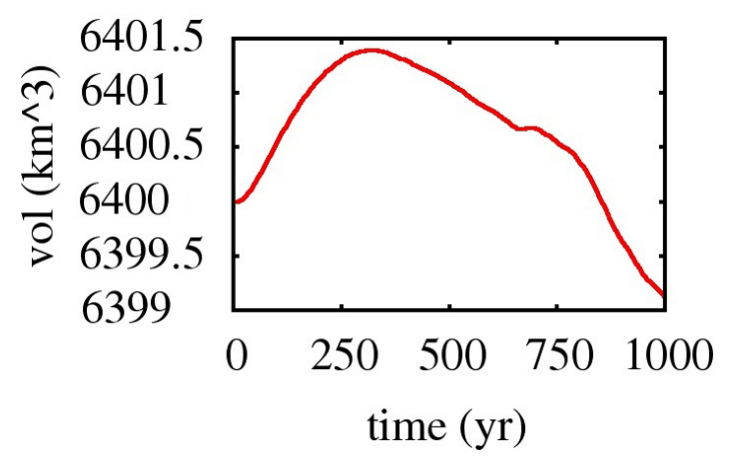

Fig. 7. Plot of the volume of the ice sheet at each of the time steps for the time-dependent ice-sheet flow experiment using the grid of resolution $80 \times 80 \times 20$.

and Eq. (44) at the top surface of the ice sheet at times $t=0$, 100 , and $1000 \mathrm{yr}$. Figure 3 shows results along the cross section at $y=L / 4$ at the same times. Because the thickness is not uniform at the beginning of the simulation, the velocity and free surface co-evolve over the length of the simulation in order to increase or decrease the surface elevation, eventually leading to a uniform ice thickness everywhere in the domain. At the steady state, the ice flow is almost uniform horizontally but layered in the vertical.

\subsection{Convergence of the Stokes solver for the ice-sheet dynamics}

We first test the convergence of the Stokes solver for the ice-sheet dynamics using the manufactured analytical solution at the initial time $t=0$. Four sets of tetrahedral grids with uniform refinement (starting with a $20 \times 20 \times 5$ structured prismatic mesh) were used, with a maximum number of degrees of freedom (DOF) of 25985444 at the finest grid resolution $(160 \times 160 \times 40)$. The viscosity $\mu$ in this manufactured solution could be huge or even infinite at some locations; in our experiments we remove the viscosity singularity problem by setting $\mu$ to be a very large number, e.g., $10^{10}$, at those points; this practical treatment may result in some additional but small numerical errors near the singularity points. Numerical results from these tests are presented in Table 1 . In Table 1 , the error measure is the $L^{2}$ norm of the solution absolute error, and the units are $\mathrm{m} \mathrm{a}^{-1} \mathrm{~km}^{3}$ for velocity and $10^{5} \mathrm{~Pa} \mathrm{~km}^{3}$ for pressure. Our approximate solutions clearly match the analytical solution well, i.e., it is easy to observe the errors reducing with grid refinement. Suppose that the error reduces with the grid refinement as $\left\|u_{\mathrm{h}}-u\right\|_{L^{2}}=O\left(h^{r}\right)$, where $h$ is the maximum element size of the grid and $u_{\mathrm{h}}$ is the numerical solution on such a grid, then $r$ is called the rate of convergence. If the convergence rates in the Table 1 are calculated between consecutive pairs of grids, e.g., the errors on a series of uniformly refined grids are $e_{1}, e_{2}, \ldots, e_{K}$, then the corresponding convergence rates are $\log _{2}\left(\frac{e_{i-1}}{e_{i}}\right), i=2, \ldots, K$, respectively. The rates of con- vergence shown in Table 1 are larger than 2.33 for the velocity and 1.51 for the pressure. The errors of the finite element method with P2-P1 element pairs for solving constantviscosity Stokes problems are theoretically $O\left(h^{3}\right)$ for velocity and $O\left(h^{2}\right)$ for pressure. The ice-sheet Stokes model is a nonlinear, variable-viscosity Stokes problem and in our tests the obtained convergence rates are similar or slightly lower than the optimal ones for the constant-viscosity Stokes problem. Error distributions of the numerical simulations (using the grid of resolution $160 \times 160 \times 40$ ) of the three velocity components and the pressure on the top surface and along the cross section at $y=L / 4$ are presented in Fig. 4 . We observe that the error of the simulation results is relatively large along the lines $x+y=L / 2, x+y=L$, and $x+y=3 L / 2$, near which the viscosity $\mu$ of the manufactured solution has quite sharp changes.

\subsection{Simulation of time-dependent ice-sheet flows}

To simulate time-dependent ice-sheet flow from $t=0$ to $t=1000 \mathrm{yr}$, a grid of 768000 tetrahedra (from a $80 \times 80 \times 20$ structured prismatic mesh) was used, resulting in 3296724 DOFs. We divided the period $[0,1000]$ uniformly with a time step $\Delta t=5 \mathrm{yr}$ to obtain a set of time steps $\left\{t_{k}\right\}_{k=0}^{200}$. At each time step $t_{k}$, with $0 \leq k<200$, we compute the ice velocity from the numerical model and update the ice thickness at time $t_{k+1}$ according to the free surface Eq. (7), using an explicit finite element discretization scheme (Leng et al., 2012). We specially remark that for this manufactured solution, when the ice sheet gets closer to the steady state, the viscosity $\mu$ has overall stronger singularity which makes computational simulations more difficult. Thus, it is also worth pointing out that for this example some smoothing techniques as a post processing could be applied to the velocity on the top surface around places where the viscosity has sharp changes (it often causes large discontinuity in the approximate solution), in order to improve accuracy in updating the elevation of the ice-sheet top surface through numerical solution of Eq. (7). We applied the Gaussian smoothing in our simulations.

Figure 5 plots the average and maximum absolute errors at each of the time steps in our time-dependent ice-sheet flow experiment, and Fig. 6 presents distribution of the errors of the computed top surface elevation at 100,300, and $1000 \mathrm{yr}$. The error of the modeled ice-sheet top surface is accumulating linearly as time goes on as shown, the average absolute error is about $1 \mathrm{~m}$ at $100 \mathrm{yr}, 2.2 \mathrm{~m}$ at $300 \mathrm{yr}$, and $8 \mathrm{~m}$ at $1000 \mathrm{yr}$, and the corresponding maximum absolute error is about $2.5 \mathrm{~m}, 8 \mathrm{~m}$, and $30 \mathrm{~m}$, respectively. Another fact we observe is that although the analytical solution reaches the geometrically steady state as $t \rightarrow \infty$, the approximate solutions computed by the numerical model continue to accumulate errors as time goes on. We believe that it is partially because the errors in the velocity solution and the surface elevation solution keep on interacting with each other and 
Table 1. $L^{2}$ errors in the velocity and pressure simulated by the parallel high-order accurate, finite element Stokes ice-sheet model at the initial state $t=0$.

\begin{tabular}{llllll}
\hline Mesh & DOF & $\begin{array}{l}\text { Velo. } \\
\text { error }\end{array}$ & $\begin{array}{l}\text { Conv. } \\
\text { rate }\end{array}$ & $\begin{array}{l}\text { Pres. } \\
\text { error }\end{array}$ & $\begin{array}{l}\text { Conv. } \\
\text { rate }\end{array}$ \\
\hline $20 \times 20 \times 5$ & 56184 & $2.67 \times 10^{1}$ & - & $1.91 \times 10^{1}$ & - \\
$40 \times 40 \times 10$ & 424364 & $4.00 \times 10^{0}$ & 2.74 & $6.70 \times 10^{0}$ & 1.51 \\
$80 \times 80 \times 20$ & 3296724 & $3.16 \times 10^{-1}$ & 3.66 & $1.82 \times 10^{-1}$ & 1.88 \\
$160 \times 160 \times 40$ & 25985444 & $6.30 \times 10^{-2}$ & 2.33 & $5.66 \times 10^{-2}$ & 1.68 \\
\hline
\end{tabular}

prevent the approximate solution from reaching a numerically steady state. Figure 7 plots the volume of the ice sheet at each of the time steps. Theoretically, the volume remains at the constant value $6400 \mathrm{~km}^{3}$ along the time. However, in the numerical simulations, because the incompressibility condition is not exactly satisfied in each element by the finite element Stokes solver nor is the mass conserved by the finite element solver for updating the ice-sheet thickness, the total volume of the ice sheet shows very slight variations having a maximum relative value of $0.00022\left(-0.8 \sim 1.4 \mathrm{~km}^{3}\right.$ out of $6400 \mathrm{~km}^{3}$ ) over the whole simulation. This could be another cause of the growing simulation errors. Using refined grids can result in better results. Overall, the modeled icesheet top surface in the whole simulation agrees well with the analytical solution $s(x, y, t)$, which demonstrates that our computational finite element Stokes ice-sheet flow model is quite accurate for this sample problem.

\section{Conclusions}

In this paper, following the ideas in Sargent and Fastook (2010), we derived manufactured solutions for isothermal, three-dimensional, nonlinear Stokes flow ice-sheet models. Their applicability for verifying numerical models was demonstrated through comparison to outputs from the finite element flow model of Leng et al. (2012). The high-order accurate, finite element Stokes ice dynamics solver was shown to be accurate and to posses good convergence rates with finer grid resolution. The solutions derived and demonstrated here should be of general use by the ice-sheet modeling community for the verification of nonlinear Stokes flow glacier and ice-sheet models.

In practice, the Stokes dynamics solver is coupled to an energy equation for the ice-sheet temperature. The interplay between velocities, pressures, and temperatures have important effects on evolving ice sheets. Thus, it would certainly be worthwhile (although certainly not easy) to derive analytical solutions for the coupled Stokes, energy, and height evolution system governing the thermo-mechanics of ice sheets and to use such solutions to verify simulation codes for this system.

\section{Supplementary material related to this article is available online at: http://www.the-cryosphere.net/7/19/ 2013/tc-7-19-2013-supplement.zip.}

Acknowledgements. This work was partially supported by the US Department of Energy, Office of Science, Advanced Scientific Computing Research and Biological and Environmental Research programs through DE-FG02-07ER64431, DE-FG02-07ER64432, DOE 07SCPF152, and the Scientific Discovery through Advanced Computing (SciDAC) project "PISCEES", and by the US National Science Foundation under the grant number DMS-1215659. We also would like to thank the referees and the editor for their insightful comments which led to substantial improvements in the paper.

Edited by: O. Gagliardini

\section{References}

Bahr, D.: Analytical modeling of glacier dynamics, Math. Geology, 28, 229-251, 1996.

Bueler, E., Lingle, C., Kallen-Brown, J., Covey, D., and Bowman, L.: Exact solutions and verification of numerical models for isothermal ice sheets, J. Glaciol., 51, 291-306, 2005.

Bueler, E., Brown, J., and Lingle, C.: Exact solutions to the thermomechanically coupled shallow-ice approximation: effective tools for verification, J. Glaciol., 53, 499-516, 2007.

Burstedde, C., Ghattas, O., Stadler, G., Tu, T., and Wilcox, L. C.: Parallel scalable adjoint-based adaptive solution of variableviscosity Stokes flow problems, Comput. Methods Appl. Mech. Engrg., 198, 1691-1700, 2009.

Dukowicz, J., Price, S., and Lipscomb, W.: Consistent approximations and boundary conditions for ice-sheet dynamics from a principle of least action, J. Glaciol., 56, 480-496, 2010.

Gagliardini, O. and Zwinger, T.: The ISMIP-HOM benchmark experiments performed using the Finite-Element code Elmer, The Cryosphere, 2, 67-76, doi:10.5194/tc-2-67-2008, 2008.

Larour, E., Seroussi, H., Morlighem, M., and Rignot, E.: Continental scale, high order, high spatial resolution, ice sheet modeling using the Ice Sheet System Model (ISSM), J. Geophys. Res., 117, F01022, doi:10.1029/2011JF002140, 2012. 
Le Meur, E., Gagliardini, O., Zwinger, T., and Ruokolainen, J.: Glacier flow modelling: A comparison of the shallow ice approximation and the full-Stokes solution, C. R. Phys., 5, 709-722, 2004.

Leng, W., Ju, L., Gunzburger, M., Price, S., and Ringler, T.: A parallel high-order accurate finite element Stokes ice sheet model, J. Geophys. Res., 117, F01001, doi:10.1029/2011JF001962, 2012.

Leysinger Vieli, G. and Gudmunsson, G.: On estimating length fluctuations of glaciers caused by changes in climatic forcing, J. Geophys. Res., 109, F01007, doi:10.1029/2003JF000027, 2004.

Morlighem, M., Rignot, E., Seroussi, H., Larour, E., Dhia, H., and Aubry, D.: Spatial patterns of basal drag inferred using control methods for a full-Stokes and simpler models for Pine Island Glacier, West Antarctica, J. Geophys. Res., 37, L14502, doi:10.1029/2010GL043853, 2010.

Nye, J.: The distribution of stress and velocity in glaciers and ice sheets, Proc. R. Soc. London Ser. A, 239, 113-133, 1957.

Pattyn, F.: A new 3D higher-order thermomechanical ice-sheet model: Basic sensitivity, ice-stream development and ice flow across subglacial lakes, J. Geophys. Res., 108, 2382, doi:10.1029/2002JB002329, 2003.
Pattyn, F., Perichon, L., Aschwanden, A., Breuer, B., de Smedt, B., Gagliardini, O., Gudmundsson, G. H., Hindmarsh, R. C. A., Hubbard, A., Johnson, J. V., Kleiner, T., Konovalov, Y., Martin, C., Payne, A. J., Pollard, D., Price, S., Rückamp, M., Saito, F., Souc(ek, O., Sugiyama, S., and Zwinger, T.: Benchmark experiments for higher-order and full-Stokes ice sheet models (ISMIPHOM), The Cryosphere, 2, 95-108, doi:10.5194/tc-2-95-2008, 2008.

Sargent, A. and Fastook, J. L.: Manufactured analytical solutions for isothermal full-Stokes ice sheet models, The Cryosphere, 4, 285-311, doi:10.5194/tc-4-285-2010, 2010.

Schoof, C. and Hindmarsh, R.: Thin-Film Flows with Wall Slip: An Asymptotic Analysis of Higher Order Glacier Flow Models, Quat. J. Mech. Appl. Maths., 63, 73-114, 2010.

Seroussi, H., Ben Dhia, H., Morlighem, M., Larour, E., Rignot, E., and Aubry, D.: Coupling ice flow models of varying orders of complexity with the tiling method, J. Glaciol., 58, 776-786, 2012.

Zhang, H., Ju, L., Gunzburger, M., Ringler, T., and Price, S.: Coupled models and parallel simulations for three dimensional fullStokes ice sheet modeling, Numer. Math. Theo. Meth. Appl., 4, 359-381, 2011. 